\title{
A Structured Analytical Approach to Handwritten Marathi vowels Recognition
}

\author{
Nilima P. Patil K. P. Adhiya Surendra P. Ramteke \\ SSBT'S College of Engineering \& Technology Bambhori, Jalgaon
}

\begin{abstract}
:
In OCR domain, it is now widely accepted that a single feature extraction method and single classification algorithm can't yields better performance rate. It is therefore, a compound feature extraction approach based on structural analysis for recognition of offline handwritten Marathi vowels is proposed. Though, Moment invariant technique is well experimented by many researchers, an attempt is made to enhance the existing results by extracting various supportive features like affine invariant moments, image thinning, structuring the image in box format, etc. These features are independent of size, slant, orientation, translation and other variations in handwritten characters. 5 samples of each vowel from 25 different people have been sampled and database was prepared. After segmentation, an individual image is resized to 50X50. 33 different features were evaluated for each character. The Fuzzy Gaussian Membership Function has been adopted for classification. The main objective of the paper is to test the possibility of using the MI, AMI combination of both for recognition of Handwritten Marathi vowels. The results show the satisfactory performance rate.
\end{abstract}

Keywords: Feature Extraction, Moment invariants, OCR, Gaussian Function,

\section{INTRODUCTION}

OCR has been extensively researched for more than four decades. With the advent of digital computers, many researchers and engineers have been involved in this important area. Handwritten Character recognition is an exigent task due to the restricted shape variation, different script style \& different kind of noise that breaks the strokes in number or changes their topology [1,2]. As handwriting varies when person write a same character twice, one can expect enormous dissimilarity among people. These are the reason that made researchers to find techniques that will improve the knack of computers to characterize and recognize handwritten characters [3, 4]. OCR is not only a new developing area due to many potential applications such as bank check processing, postal mail sorting, automatic reading of tax forms, and reading various handwritten and printed text and non-text documents[5,6]. It is also a benchmark for testing and verifying new pattern recognition theories and algorithms. It can be used as a reading machine for the visually handicapped when interfaced with a voice synthesizer $[7,8,9]$.

Among the various phases of an OCR/HCR, the feature extraction phase is quite important as a set of useful properties of a character available as an image is defined and extracted during this phase. More relevant is the feature extraction method(s) used for discrimination $[10,11]$. In this paper, we have studied, implemented and compared the performance of Moment Invariants based methodologies. 5 samples of each Marathi vowels from 25 different people have been sampled and database was prepared. After segmentation, an individual image is normalized to 50X50 pixel sizes. Seven moment invariants (MIs), Four Affine moment invariants (AMIs) \& combination of both are evaluated for each character. Three kinds of moment invariants based features are extracted as follows

i. Direct feature extraction.

ii. Thinning process

iii.Box approach.

In each case we got seven features so total 21 features are evaluated. Similarly, these three kinds of features are evaluated by applying affine Moment invariant methods. So total 12 features and for combination of both methods, in all 33 features are used for processing of classification.

The Fuzzy Gaussian Membership Function has been adopted for classification. The main objective of the paper is to compare the all feature extraction methods for recognition of Handwritten Marathi character independent of its Size, slant and other variations. The paper is organized as follows: In section 2, the approach of Moment Invariant, Affine moment Invariants of MI \&AMI which is adopted for feature extraction, is presented. Section 3 deals with the classification based on Fuzzy Gaussian Membership Function. The section 4 gives details of result and conclusion is discussed in section 5 .

\subsection{Devanagari Marathi Vowels}

\section{अभआइई उ ऊए ए ओओ क्रा}

Devanagari, an alphabetic script, is used by a number of Indian Languages. It was developed to write Sanskrit but was later adapted to write many other languages such as Marathi, Hindi, Konkani, Sindhi and Nepali. Many other Indian languages use close variant of this script. Marathi script has about 11 vowels and 34 consonants. In addition, there are large number of conjuncts formed by combination of consonants, their halfforms and some modifier symbols. The modifier symbols are placed either on the top, at the bottom, on the left, to the right or a combination of these. Also has a Shirorekha (a header line) runs through the entire span of word.

\section{FEATURE EXTRACTION 2.1 Moment Invariants Approach (MI)}


Moment invariants (MIs) are important tools in object recognition problems. These techniques are commonly divided into two main categories according to how they make use of the image function. In so called local approaches the objects are segmented to smaller elements and invariants are computed separately for each of them. The second category consists of the global approaches where the features are computed directly from the whole image intensity function.

The MIs were derived by means of the theory of algebraic invariants. The AMIs are invariant under general affine transformation

Regular moments are defined as [12]

$M_{\mathrm{pq}}=\iint X^{\mathrm{p}} Y^{\mathrm{q}} \mathrm{f}(\mathrm{X}, \mathrm{Y}) \mathrm{dxdy}$

Where for $\mathrm{p}, \mathrm{q}=0,1,2 \ldots$ and $\mathrm{Mpq}$ is the $(\mathrm{p}+\mathrm{q})^{\mathrm{th}}$ order moment of the continuous image function $f(x, y)$. If the image is represented by a discrete function, integrals are replaced by summations. Equation (1) can be written as follows

$$
M_{\mathrm{pq}}=\sum \sum X^{p} Y^{q} \mathrm{f}(\mathrm{X}, \mathrm{Y})
$$

The central moments of $f(x, y)$ are defined by the expression

$$
\mu_{p q}=\sum \sum\left(X-\bar{X}^{p}\left(Y-\bar{Y}^{q} \mathrm{f}(\mathrm{X}, \mathrm{Y})\right.\right.
$$

Where $\bar{X}=\left(m_{10} / m_{00}\right)$ and $\bar{Y}=\left(m_{01} / m_{00}\right)$ which are the centroid of the image.

The central moments of order up to 3 are as follows:

$$
\begin{aligned}
& \mu_{10}=0 \\
& \mu_{01}=0 \\
& \mu_{10}=m_{11}-\overline{\mathrm{Y}} m_{11} \\
& \mu_{20}=m_{20}-\overline{\mathrm{X}} m_{10} \\
& \mu_{02}=m_{02}-\overline{\mathrm{Y}} m_{01} \\
& \mu_{30}=m_{30}-3 \overline{\mathrm{Y}} m_{20}+2 \overline{\mathrm{X}}^{2} m_{10} \\
& \mu_{03}=m_{03}-3 \overline{\mathrm{Y}} m_{02}+2 \overline{\mathrm{Y}}^{2} m_{01} \\
& \mu_{21}=m_{21}-2 \overline{\mathrm{X}} m_{11}-2 \overline{\mathrm{Y}} m_{20}+2 \overline{\mathrm{X}}^{2} m_{01} \\
& \mu_{12}=m_{12}-2 \overline{\mathrm{Y}} m_{11}+\overline{\mathrm{X}} m_{02}+2 \overline{\mathrm{Y}}^{2} m_{10}
\end{aligned}
$$

The normalized central moment to shape and size of order $(p+$ q) is defined

$$
\eta_{p q}=\mu_{p q} / \mu_{00}^{\gamma} \text { for } \mathrm{p}, \mathrm{q}=0,1,2
$$

Where $\gamma=\frac{(p+q)}{2}+1$

For $(\mathrm{p}+\mathrm{q})=2,3, \ldots .$. Based on normalized central moments, a set of seven moment invariants $[12,13]$ can be derive as follows $\phi_{1}=\eta_{20}+\eta_{02}$

$\phi_{2}=\left(\eta_{20}-\eta_{02}\right)^{2}+4 \eta^{2}$

$\phi_{3}=\left(\eta_{30}-3 \eta_{12}\right)^{2}+\left(3 \eta_{21}-\eta_{03}\right)^{2}$

$\phi_{4}=\left(\eta_{30}+\eta_{12}\right)^{2}+\left(\eta_{21}+\eta_{03}\right)^{2}$

$\phi_{5}=\left(\eta_{30}-3 \eta_{12}\right)\left(\eta_{30}+\eta_{12}\right)\left[\left(\eta_{30}+\eta_{12}\right)^{2}+3\left(\eta_{21}+\right.\right.$ $\eta 032]+3 \eta 21-\eta 03 \eta 21+\eta 033 \eta 30+\eta 122-\eta 21+\eta 032$

$$
\begin{aligned}
& \phi_{6}=\left(\eta_{30}-3 \eta_{12}\right)\left[\left(\eta_{30}+\eta_{12}\right)^{2}-\left(\eta_{21}+\eta_{03}\right)^{2}\right]+4 \eta_{1}\left(\eta_{30}+\right. \\
& \eta 12 \eta 21+\eta 03 \\
& \phi_{7}=\left(3 \eta_{21}-\eta_{03}\right)\left(\eta_{30}+\eta_{12}\right)\left[\left(\eta_{30}+\eta_{12}\right)^{2}-3\left(\eta_{21}+\eta_{03}\right)^{2}\right]+ \\
& \quad\left(3 \eta_{12}-\eta_{30}\right)\left(\eta_{21}+\eta_{03}\right)\left[3\left(\eta_{30}+\eta_{12}\right)^{2}-\left(\eta_{21}+\eta_{03}\right)^{2}\right]
\end{aligned}
$$

\subsection{Affine Moment Invariant (AMIs):}

The AMIs were derived by means of the theory of algebraic invariants. The AMIs are invariant under general affine transformation

$\mathrm{u}=a_{0}+a_{1} x+a_{2} \mathrm{y}$

$v=b_{0}+b_{1} x+b_{2} y$

Where $(\mathrm{x}, \mathrm{y})$ and $(\mathrm{u}, \mathrm{v})$ are coordinates in the image plan before and after the transformation respectively. Four simplest AMIs that we have used for character recognition are listed below.[14,15]

$\mathrm{I}_{1}=1 / \mu_{00}^{4}\left(\mu_{20} \mu_{02}-\mu_{11}^{2}\right)$

$I_{2}=1 / \mu_{00}^{10}\left(\mu_{30}^{2} \mu_{03}^{2}-6 \mu_{30} \mu_{21} \mu_{12} \mu_{03}+4 \mu_{30} \mu_{12}^{3}+4 \mu_{03} \mu_{21}^{3}-\right.$ $\left.\left.3 \mu^{2}{ }_{21} \mu^{2}{ }_{12}\right)\right)$

$I_{3}=1 / \mu^{7}{ }_{00}\left(\mu_{20}\left(\mu_{21} \mu_{03}-\mu_{12}^{2}\right)-\mu_{11}\left(\mu_{30} \mu_{03}-\mu_{21} \mu_{12}\right)+\mu_{02}\left(\mu_{30} \mu_{12}-\right.\right.$ $\left.\left.\mu^{2}{ }_{21}\right)\right)$

$I_{4}=1 / \mu_{00}^{11}\left(\mu_{20}^{3} \mu_{03}^{2}-6 \mu_{20}^{2} \mu_{11} \mu_{12} \mu_{03}-6 \mu_{20}^{2} \mu_{02} \mu_{21} \mu_{03}+9 \mu_{20}^{2} \mu_{02} \mu_{12}\right.$

$+12 \mu_{20} \mu_{11}^{2} \mu_{21} \mu_{03} \quad 6 \mu_{20} \mu_{11} \mu_{02} \mu_{30} \mu_{03}-18 \mu_{20} \mu_{11} \mu_{02} \mu_{21} \mu_{12}$ $8 \mu_{11}^{3} \mu_{30} \mu_{03}-6 \mu_{20} \mu_{02}^{2} \mu_{30} \mu_{12}+9 \mu_{20} \mu^{2}{ }_{02} \mu_{21}+12 \mu_{11}^{2} \mu_{02} \mu_{30} \mu$ $\left.6 \mu_{11} \mu^{2}{ }_{02} \mu_{30} \mu_{21}+\mu^{3}{ }_{02} \mu^{2}{ }_{30}\right)$

\subsection{Thinning}

Thinning is the process to extract and apply additional constraints on the pixel elements that need to be preserved such that a linear structure of the input image will be recaptured without destroying its connectivity. In the context of image 
processing, outline from a pattern until all the lines or curves are of unit width or of single pixel wide. Thus, the reduced pattern is known as the skeleton [15]. Skeletonization or thinning is a very important preprocessing step in pattern Analysis. One major advantage of thinning is to reduction memory space required for storing the essential structural information presented in a pattern moreover it simplifies the data structure required in pattern analysis.

$$
\text { ई }
$$

Character

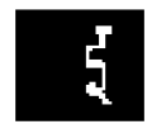

Complement

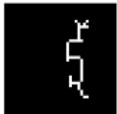

Thinning
Figure 1. Devanagari vowel through different Processes

\subsection{Box Method}

The feature of a given character is extracted in following steps 1) A given number is divided in equal blocks having (5X5) $=25$ pixels in each block.

2) A weight value is assigned to each block. It is equal to number of pixels in the block. One example is given figure 2 .

3) The weight function is normalized, with

respect to 100 .

4) The values of the weight functions along with its $(i, j)^{\text {th }}$ position are sorted in decreasing order. In the data base the numbers corresponding to the font size 10 are taken. The features in the data base are called as the known feature domains. These are $m(=10)$ known feature as standard domains in the present database with 10 features in each domain [16].
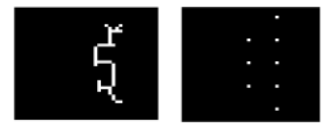

Figure 2. Character enlosed in $5 \times 5$ grid Box

\subsubsection{Comparison Process}

The ten features of a given numeral to be recognized are extracted. These features are called in unknown feature domain. A feature in known data domain corresponding to a numeral in the data base is mapped into the

unknown feature domain by using the following equations

$$
\begin{aligned}
& i^{\prime}=i_{\min }^{\prime}+\left(i_{-} i_{\min }\right)\left(\left(i_{\max }^{\prime}-i_{\min }\right) /\left(i_{\max }-i^{\prime}{ }_{\min }\right)\right) \\
& j^{\prime}=j^{\prime}{ }_{\min }+\left(j-j_{\min }\right)\left(\left(j^{\prime} \max ^{-} j_{\min }\right) /\left(j_{\max }-j^{\prime}{ }_{\min }\right)\right)
\end{aligned}
$$

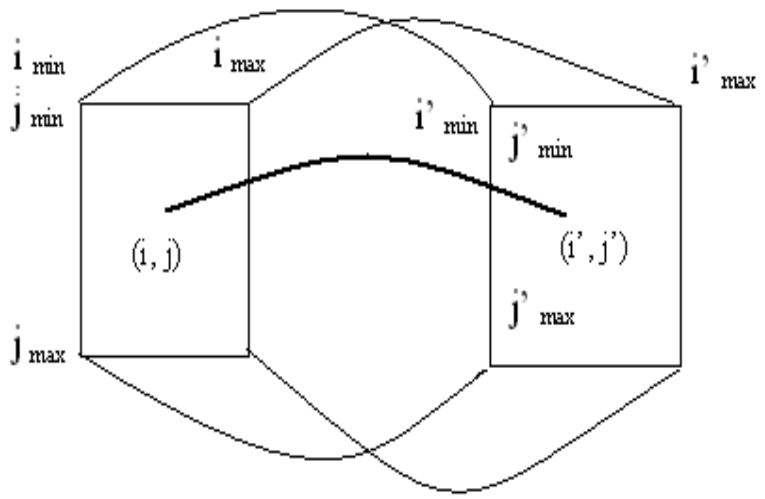

(a)

(b)

figure 3. Shows (a) the known feature from the data base (b) Feature of the unknown characters.

where $(i, j)$ of the block related to the known domain feature is mapped into (i',j') in the domain of unknown feature imin ,imax , jmin, jmax are minimum and maximum values of $i, j$ in the known domain and prime values unknown feature domain. It can be seen that the corner values of known feature domain are mapped into represent values corresponding to corners values of unknown feature domain .The new mapped block in unknown feature domain has some weight values which is estimated from the feature extracted for the unknown feature domain. The estimate is done from the nearest neighborhood value.

\section{CLASSIFICATION}

A database of numeral has been required to extract the feature, which form a template (Trained data). The reference character set has been obtained from training samples. The mean and standard deviation [14] are computed for each type of features .Therefore there are respective mean and standard deviation corresponding to each value of the character. The unknown character features available in the template and found the maximum membership value (0-1) of the character. The template consists of means $\mathrm{Mi}$ and $\sigma_{\mathrm{i}}$ for each feature and is computed as

$$
\begin{aligned}
& \text { Mean } M_{i}=\frac{1}{N} \sum I_{i(k)} \\
& \text { Std.Dev } \sigma_{i}=\sqrt{\sum\left(I_{i(k)}-M_{i}\right)^{2}}
\end{aligned}
$$

Where $\mathrm{N}_{\mathrm{i}}$ is the number of samples in $\mathrm{i}^{\text {th }}$ class and $\mathrm{I}_{\mathrm{i}(\mathrm{k})}$ Stands for the $\mathrm{k}^{\text {th }}$ feature value of reference character in the $\mathrm{i}^{\text {th }}$ class. In this work, for unknown input character $\mathrm{X}$, the corresponding features will be extracted. The Fuzzy Gaussian Membership Function [15] will be attempted to get the maximum membership value as follow

$$
\mu_{\mathrm{xi}=} \exp ^{\frac{-\left(\mathrm{x}-\mathrm{M}_{\mathrm{i}}\right)^{2}}{2 \sigma_{\mathrm{i}}^{2}}}
$$


Where $\mathrm{Xi}$ is the $\mathrm{i}^{\text {th }}$ feature of the unknown character. Let $\mathrm{Mj}(\mathrm{r})$, $\sigma j 2(r)$ belongs to the $\mathrm{r}^{\text {th }}$ reference character with $\mathrm{r}=$ character we then calculate the average membership value as

$\mu_{\mathrm{av}}(r)=\frac{1}{\mathrm{C}} \sum_{\mathrm{j}=1}^{\mathrm{c}} \exp \frac{\frac{-\left(\mathrm{X}-\mathrm{M}_{\mathrm{i}}\right)^{2}}{2 \sigma_{\mathrm{i}}^{2}}}{}$

Where $\mathrm{X} \varepsilon \mathrm{r}$ if $\mu_{\mathrm{av}}(\mathrm{r})$ is the maximum for $\mathrm{r}=$ characters

\section{RESULTS}

We applied our scheme on 1100 character images obtained from different individual. The data set was containing varieties of writing styles. The experimental work has been implemented with the help of MATLAB 7.5.0 (R2007b ) Tools .In our experiment 75 images of each character are used for training and 100 images are used for testing from the database .Following table1 shows the \% recognition rate of MIs technique is 75 $\%$,AMI gives $89.09 \%$ and combination approach of MIs \& AMIs gives $52.90 \%$ for the different devanagari Marathi vowels. Graph1 shows comparative study of all three methods attempted for Marathi vowels recognition.

Table: Recognition rate of Devanagari vowels

\begin{tabular}{|c|c|c|c|c|c|}
\hline $\begin{array}{c}\text { Devanagari } \\
\text { Character }\end{array}$ & $\begin{array}{l}\text { English } \\
\text { Character }\end{array}$ & $\begin{array}{l}\text { Total } \\
\text { Images }\end{array}$ & MIs & AMI & $\begin{array}{c}\text { MIs } \\
+ \text { AMI }\end{array}$ \\
\hline अ & A & 100 & 76 & 90 & 49 \\
\hline इ & $\mathrm{E}$ & 100 & 53 & 89 & 52 \\
\hline 3 & $\mathrm{U}$ & 100 & 88 & 87 & 57 \\
\hline ऊ & $\mathrm{OO}$ & 100 & 75 & 85 & 53 \\
\hline ए & $\mathrm{AE}$ & 100 & 80 & 89 & 54 \\
\hline ॠ & $\mathrm{HRU}$ & 100 & 75 & 82 & 56 \\
\hline आ & AA & 100 & 80 & 85 & 53 \\
\hline ई & $\mathrm{EE}$ & 100 & 66 & 91 & 54 \\
\hline ऐ & $\mathrm{AE}$ & 100 & 91 & 95 & 55 \\
\hline ओ & $\mathrm{O}$ & 100 & 88 & 97 & 53 \\
\hline औ & $\mathrm{AU}$ & 100 & 53 & 90 & 46 \\
\hline \multicolumn{3}{|c|}{ Recognition Rate in \% } & 75 & 89.09 & 52.90 \\
\hline
\end{tabular}

MIs-Moment Invariant , AMIs-Affine Moment Invariant

Graph 1: Comparative study of different methods of handwritten Marathi vowels recognition

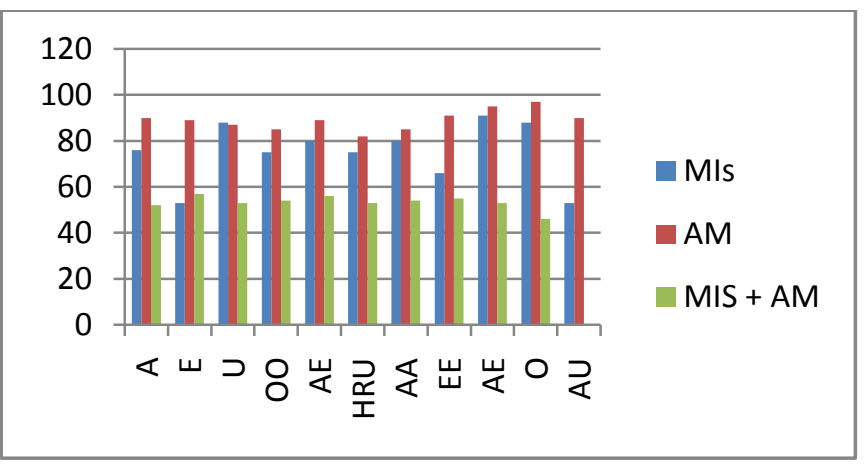

\section{CONCLUSION}

In this paper we have proposed a Fuzzy Gaussian Membership Function based approach to recognition of handwritten Devanagari words. The results of our approach are promising. The accuracy reported in this paper is based on one step recognition around $89.09 \%$ for AMI method. We can apply two stage recognition approaches to improve the performance of the scheme. A point to be noted here is that we have not applied any post processing step. Post processing can definitely improve the performance which we will undertake in our future work.

\section{REFERENCES}

[1] N.Arica, F.T.Y. Vural, "An overview of Character recognition focused on offline Handwriting", IEEE trans on system ,man,cybernatics-Partc, vol 31,N0.2(2001).

[2] Ovind Trier, Anil Jain and Torfinn taxt," A feature extraction methods for character recognition-A survey", pattern recognition, vol 29, no-4, and pp 641-662, 1996.

[3] U.Pal and B.B. Chaudhuri," An improved document skew angle estimation techniques", Pattern Recognition Letters 17:899-904, 1996.

[4] B.B. Chaudhruri and U.Pal, "A complete printed OCR", Pattern Recognition, (5):531-549, 1998.

[5] Rejean Plamondon and Sargur N. Srihari, "On-Line and Off-Line Handwritten Recognition" A Comprehensive Survey", IEEE Pattern Analysis and Machine Intelligence, Vol 22, No. 1, January 2000.

[6] U. Pal, T. Wakabayashi, F. Kimura, "Comparative Study of Devnagari Handwritten Character Recognition using Different Feature and Classifiers", 10th International Conference on Document Analysis and Recognition 2009.

[7] Zhiyi Zhang, Lianwen Jin, Kai Ding, Xue Gao,"CharacterSIFT: a novel feature for offline handwritten Chinese character Recognition" 10th International Conference on Document Analysis and recognition, 2009

[8] T.V.Aswin and P S Sastry, "A font and size-independent OCR system for printed Kannada documents using support vector machines", Sadhana Vol.27.part I, pp.35-58, February 2002.

[9] T.V.Aswin, "A font independent OCR for printed Kannada using SVM", Master thesis, Indian Institute of Science, Bangalore, 2000.

[10] Veena Bansal and R. M. K. Sinha, "A Devanagari OCR and A Brief Overview of OCR Research for Indian Scripts”, Proceedings of STRANS01, IIT Kanpur 2001

[11] V. D. Bhagile, R. J. Ramteke, S. C. Mehrotra, "A Block Based Approach To Recognition of Printed Numerals 
Irrespective of Font Size”, at National Conference on Emerging Trends in Information Technology (eIT 2006), VIIT, Baramati, Pune, 29-30, Sept. 2006.

[12] R.J. Ramteke, P.D.Borkar, S.C.Mehrotra, "Recognition of Isolated Marathi Handwritten Numerals: An Invariant Moments Approach", pp.482-489, Proceedings of the International Conference on Cognition and Recognition, Dec-2005

[13] Jan Fluseer,2000. On the independence of rotation moment invariants, pattern recognition 33(2000) 14051410

[14] .J.Ramteke, "Invariant moment based feature extraction for handwritten devanagari vowels recognition",
International Journal of computer application (09758887), Volume 1-No.18, 2010.

[15] Flusser and suk "Affine moment invariants: A new tool for character recognition "pattern recognition letters, vol. 15, pp. 433-436, 1994.

[16] L.Heuttte, T.Paquet, J.V. Moreau, .Lecourtier, C.Oliver, (1998), "A structural /Statistical feature based vector for handwritten character recognition", Pattern Recognition Letters 19,pp.629-64117.

[17] R. C. Gonzalez, R. E. Woods, "Digital Image Processing using MATLAB", Pearson Education, 2004. 